\title{
THE EFFECT OF GeOMETRICAL PARAMETERS ON HEAT TRANSFER AND HYDRO DYNAMICAL CHARACTERISTICS OF HELICAL EXCHANGER
}

\author{
Mir Hatef Seyyedvalilu ${ }^{1}$ and S.F.Ranjbar ${ }^{2}$ \\ ${ }^{1}$ MSc, Mechanical Engineering Faculty, University of Tabriz, Iran \\ ${ }^{2}$ Assistant Professor of Mechanical Engineering, University of Tabriz, Tabriz, Iran
}

\begin{abstract}
Compact size and high heat transfer coefficient of helical coil heat exchangers causes them to have an important role in various industrial applications. This paper investigate numerically on the influence of different parameters such as coil radius, coil pitch and diameter of tube on the hydrodynamic and heat transfer characteristics of helical double tube heat exchangers using the CFD software which is based on the principles of heat transfer, fluid mechanics and thermodynamics. The results indicated that heat transfer augmentation occurs by increasing of the inner Dean Number, inner tube diameter, curvature ratio and by the reduction of the pitch of heat exchanger coil. By increasing the radius of coils, the secondary flow effects due to centrifugal forces diminishes and flow of fluid through the coils tends to flow in a straight path and as a result, the friction coefficient decreases consequently.
\end{abstract}

\section{KEYWORDS}

Helical double tube heat exchanger; pressure drop; Dean Number; overall heat transfer coefficient

\section{INTRODUCTION}

The role of heat transfer enhancement is so important in size reduction, thermodynamic efficiency enhancement, and pumping power reduction. Therefore, for heat transfer rate increasing, different methods can be applied. Surveying of heat exchangers and heat transfer issues and it's enhancement through inactive methods, requires devising a new scheme. In double tube helical heat exchangers, due to the curvature of tubes and exertion of centrifugal force on fluid flow, the secondary flow motion was generated which improves heat transfer coefficient substantially. In the survey of performance of these equipment, several parameters were taken into account such as Dean Number, curvature ratio, tube diameter, and coil pitch. Although these methods increase the heat transfer between two or more fluids, they create a pressure drop through the path of flow. Therefore, it can be said that, pressure drop is the cost of increasing the heat transfer rate in the heat exchangers.

In recent years, many of researchers worked on the heat transfer and hydrodynamic characteristics of fluid in double tube heat exchangers. Rennie and Raghavan [1] studied numerically on the heat transfer characteristics of a double tube heat exchanger. They proved that, flow in the inner tube, is the limiting factor of overall heat transfer coefficient of heat exchanger and while stabilizing other parameters, the overall heat transfer coefficient will increase. Rennie and Raghavan [2] studied the heat transfer characteristics of helical double tube heat exchanger to determine the effect of fluid thermal properties on heat transfer. They demonstrated that in lower Dean Number, Nusselt number was more affected by Prandtel number than in higher Dean 
Number. Kumar et al. [3] experimentally and numerically studied heat transfer coefficients of helical double tube heat exchanger. They pointed out that overall heat transfer coefficient of heat exchanger increases in the presence of constant mass flow rate within annulus by the increase of Dean Number of inner pipe, and vice versa. Lin and Ebadian [4] experimentally studied on the hydrodynamic characteristics of R-134a in helical tubes. Results indicated that Nusselt Number of refrigerant in lower saturation temperatures was so high and increased with increasing of mass flow rates of refrigerant and cooling water. They also indicated that flow of refrigerant in annulus can lead to higher-pressure drop than inner tube. Numerical study of helical double pipe heat exchanger was performed by Kumar et al. [5]. They obtained overall heat transfer coefficient of heat exchanger for different flow rates in inner and outer tubes. Garrido et al. [6] developed a numerical model for heat transfer and dynamic behavior of fluid in helical double pipe evaporator. Inner pipe pressure and temperature of the annulus are two effective parameters in determination of increase of outlet temperature. Xiaowen and Lee [7] experimentally surveyed performance of Window Air Conditioner (WAC) by using of helical double pipe heat exchanger for preheating of hot water. Results indicated that the performance of WAC increased by the use of heat recovery system. Xin et al. [8] studied single-phase flow in helical double tube heat exchanger in horizontal and vertical arrangements. In their work, influence of coil geometry, flow rate of air and water on pressure drop of single-phase flow was surveyed. Petrakisc and Karahalios [9] studied viscous fluid flow in coils of double tube while pressure gradient along the axis reduces exponentially with time. In another work [10], they obtained numerical solution of incompressible viscous fluid flow equation for water flowing in a curved double tube with circular cross section. In this investigation, it was indicated that in small radius of core, change of Dean Number has considerable effect on fluid's properties whereas it was not observed in large radius. Pressure drop characteristics of R-134a in helical double tube were surveyed by Han et al. [11]. Beigzadeh and Rahimi [12] presented a model by the use of artificial neural network to calculate heat transfer coefficient and friction factor in helical tubes. They indicated that the neural network can be very useful in the prediction of the amount of heat transfer and flow characteristics of helical heat exchanger. Di liberto and Ciofalo [13] studied the heat transfer of turbulent flow in curved tubes by numerical simulation. They also used this method to fully survey of developed turbulent flow in curved tubes. Results of this study indicated that in the curved tubes, temperature fluctuations in the outer regions are more drastic than the other regions. Gabriela and Angel Huminic [14] used analysed three dimensionally nano-fluids to study the heat transfer characteristics of helical double tube heat exchanger. They found that Nano fluids increase fluid thermal conductivity and heat transfer.

Majority of the performed studies in the field of analysing and testing of helical heat exchangers, are limited to a single helical coil, whereas this study deals with helical heat exchangers with several coils that were analysed and their thermally development status was investigated and also tried to investigate the effect of geometrical properties on heat transfer characteristics.

\section{SOLVING APPROACH}

\subsection{Characteristics of helical coils}

Stretch of the flow through the coils, is one of the most important characteristics of helical heat exchangers. According to Figure1 (a), the centrifugal forces are created because of this stretch of flow which leads to secondary flow. In figure 1(a), it is observed that maximum velocity is occurred in central region of the inner tube because this region has maximum distance from walls and thus the boundary layer. 


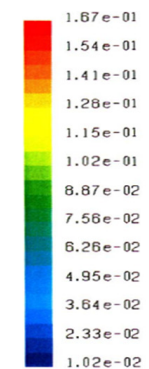

Figure 1. Schematic of inlet and outlet vectors of parallel flow in helical double pipe heat exchanger.

As Reynolds number is used to analysis of flow in straight tubes, Dean Number $(D e)$ is used for analysis of helical tubes which is defined as follows [15]:

$$
D e_{i n}=\left(\frac{V d_{i}}{v}\right)\left(\frac{d_{i}}{2 R}\right)^{1 / 2}
$$

Where, $d_{i}$ is the diameter of the inner tube, $V$ is the average velocity of the flow and $R$ is the radius of the coils. As helical double tube heat exchangers are used in laminar flow, thus in this work, laminar flow ranges were chosen for flow rates. Therefore, to know whether flow is laminar or turbulent, the obtained Reynolds number can be compared with critical number [16]:

$$
\operatorname{Re}_{\mathrm{cr}}=2100\left(1+12 \delta^{1 / 2}\right)
$$

Where $\delta$ is curvature ratio and defined as $\delta=\left(\frac{d}{2 R}\right)$.

\subsection{Problem description}

The model used in this analysis is the experimental model presented by Rennie and Raghavan [15] (Figure2). The model is a double pipe heat exchanger with pipes made of copper that $60^{\circ} \mathrm{C}$ water, as hot fluid, flows through inner tube. On the other hand $22.1^{\circ} \mathrm{C}$ Water flows in annulus pipe as cooling fluid, and in the same direction with hot water. The geometrical properties of the model can be seen in Figure 2. The thickness of both tubes is $0.8 \mathrm{~mm}$ and each coil with annulus diameter of $23.59 \mathrm{~mm}$, has wrapped up completely.

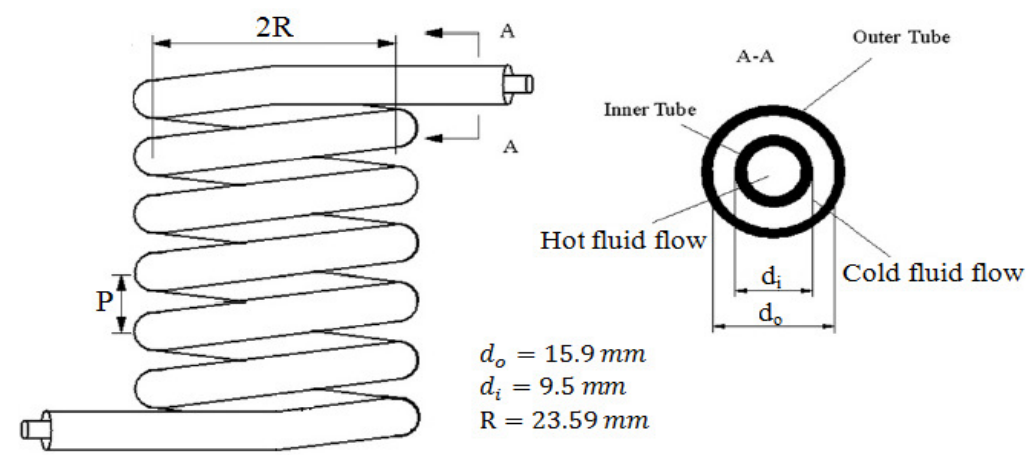

Figure 2. Schematic of used model 


\subsection{Mesh generation process}

Choosing a suitable mesh generation technique requires a complete examination and consideration of criteria, which many papers were published with regard to this category, among which Xavier et al. [17] can be mentioned. They suggested Tetrahedral meshing because of its short-term generation and stabilization. For this reason, as can be seen from Figures 1(b) and 3, Structured Tetrahedral mesh was generated, in Gambit software, for this model.

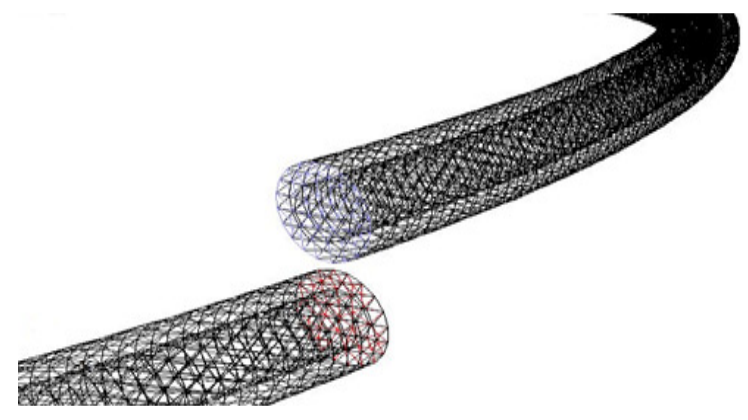

Figure 3. Grids used in the analysis.

According to the results of mesh generation process, a model with 75664 grids is chosen for the base of present work.

\subsection{Governing equations}

Fluid properties were assumed variable [18] for a realistic survey. Following Polynomial functions (Eqs. (3)- (6)) were programmed in FLUENT For modelling temperature dependent properties. The governing equations are solved with the given fluid properties.

$$
\begin{aligned}
& \mu(T)=2.1897 e-11 T^{4}-3.055 e-8 T^{3}+1.6028 e-5 T^{2}-0.0037524 T+0.33158 \\
& \rho(T)=-1.5629 e-5 T^{3}+0.011778 T^{2}-3.0726 T+1227.8 \\
& k(T)=1.5362 e-8 T^{3}-2.261 e-5 T^{2}-0.010879 T-1.0294 \\
& C_{p}(T)=1.1105 e-5 T^{3}-0.0031078 T^{2}-1.478 T+4632
\end{aligned}
$$

In equations (3) to (6), $\mu$ is the dynamic viscosity, $\rho$ is the density of fluid, $\mathrm{k}$ is the thermal conductivity of the flow and $C_{p}$ is the specific heat of fluid. All of physical phenomena, including fluid flow and heat transfer, can be analysed by using of continuity (7), momentum (8) and energy (9) equations:

$$
\begin{aligned}
& \frac{\partial u_{i}}{\partial x_{i}}=0 \\
& \frac{\partial\left(u_{i} u_{j}\right)}{\partial x_{i}}=\frac{\partial}{\partial x_{i}}\left(v \frac{\partial u_{j}}{\partial x_{i}}\right)-\frac{1}{\rho} \frac{\partial P}{\partial x_{j}} \\
& \frac{\partial\left(u_{i} T\right)}{\partial x_{i}}=\frac{\partial}{\partial x_{i}}\left(\alpha \frac{\partial T}{\partial x_{i}}\right)
\end{aligned}
$$

$u_{i}$ and $u_{j}$ is velocity in $x, y$ and $z$ direction and $T$ is temperature. 


\subsection{Boundary conditions}

Inlet boundary condition, which was defined for both inner tube and annulus, is in terms of velocity and defined as velocity inlet, because the entrance velocities of the flow are known in this case. In this work, pressure outlet is used for outlet boundary condition, and assumed that, the outlet pressure is equal to the ambient pressure. In the modelled helical double tube heat exchanger, there are two walls. For an outer tube wall, adiabatic boundary condition was assumed with zero heat flux, and for inner tube, bilateral wall was chosen. It should be noted that the heat transfer is possible on both sides.

\subsection{Calculation of heat transfer coefficient}

Overall heat transfer coefficient is obtained by using of given inlet and outlet temperatures, and following equations (10-11):

$$
\begin{aligned}
& U=\frac{\dot{q}}{A_{o} \Delta T_{\text {LMTD }}} \\
& \Delta T_{\text {LMTD }}=\frac{\Delta T_{2}-\Delta T_{1}}{\ln \left(\Delta T_{2} / \Delta T_{1}\right)}
\end{aligned}
$$

$T_{h, i}$ and $T_{h, o}$ are hot fluids inlet and outlet temperatures relatively.

\subsection{Model validation}

In this work, the numerical method was used to investigate the model. It should be noted that the numerical method is not reliable enough to get the trusted results. Hence, at the first, the results of numerical work compared with experimental ones at the literature [15]. In Figure 4 and 5, results of problem's solution and experimental work [15] for parallel and laminar flow are compared. The problem is solved for annulus mass flow rates of 100,300 and $500 \mathrm{~cm}^{3} / \mathrm{min}$ and different Dean Numbers for the inner tube. Overall heat transfer coefficient is obtained by using of equations 10 and 11. Both of the figures 4 and 5 show good agreement between numerical results and experimental work.

As regards, the numerical method is not a trusted one to study the effects of different parameters, hence the results get from this method have some differences with the actual results, because various factors such as sediment factor participated in the calculation of heat exchangers and it may not be considered in the numerical analysis. From figure 4, it can be noticed that, for the overall heat transfer coefficient, the average error related to the inner Dean number of 208.1 is equal to 2.3 percent; hence, this inner Dean number was chosen to be the basis of future heat transfer calculations in the current study. On the other hand, from figure5 the comparison between the results of numerical and experimental work, show the best agreement in hydrodynamical properties such as friction factor.

The experimental results of friction factor were given from the equation 14 which was defined and reached by Colorado et al. [19]: 


$$
\frac{f_{c}}{f_{S}}=\left[1+0.14\left(\frac{d}{2 R}\right)^{0.97} \mathrm{Re}\right]^{i}
$$

W

here $i=1-0.644\left(\frac{d}{2 R}\right)^{0.312}$ and for $R e<2300$ the straight tube's friction factor is equal to $f_{s}=\frac{64}{\operatorname{Re}}$.

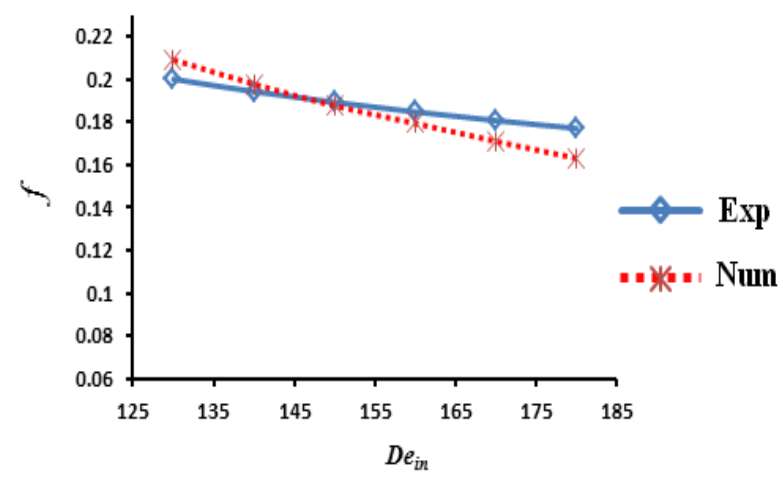

Figure 5. Comparison between numerical and experimental [19] results for inner friction factor.

\section{Results and discussion}

For augmentation of heat transfer between two fluids, secondary flow plays the main role in helical heat exchangers. Therefore, in this study, the effects of several geometrical parameters on heat transfer characteristics were surveyed. Obtained results can be presented as curvature ratio effect, increase of loop pitch of heat exchanger, tube diameters and influence of number of loops on heat transfer characteristics.

\subsection{Comparison of velocity and temperature contour in straight and helical double tube heat exchanger}

In Figures 6, $\mathrm{x}$ is the distance from the inlet of heat exchanger. As winding of the loop is $360^{\circ}, \mathrm{x}$ values of $0.37,0.74$ and $1.11 \mathrm{~m}$ represent angles of $90^{\circ}, 180^{\circ}$ and $270^{\circ}$, respectively. With comparing of these three contours, it can be seen that, in helical double tube heat exchanger, flow becomes disordered.

Because according to Figure 1(a,b), in helical double tube heat exchanger, flow is stretched and the stretch in all of three cases is from inner wall towards outer wall of heat exchanger. This stretch can be attributed to centrifugal forces in helical double tube heat exchanger. Secondary flow is a result of centrifugal forces.

As seen from figures 6 and 7, due to the presence of centrifugal forces, the temperature contours, like the velocity contours, tend to stretch towards the outer wall of helical heat exchanger. But these contours in straight tube heat exchanger are symmetric. 

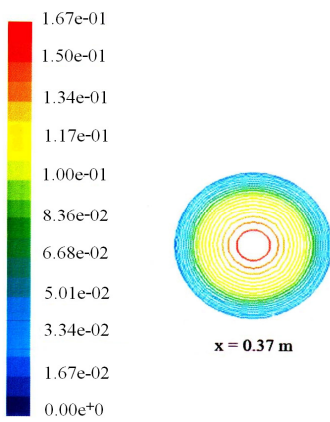

$\mathrm{x}=0.37 \mathrm{~m}$

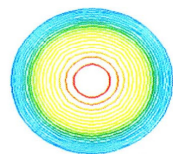

$\mathbf{x}=\mathbf{0 . 7 4} \mathbf{m}$

(a)
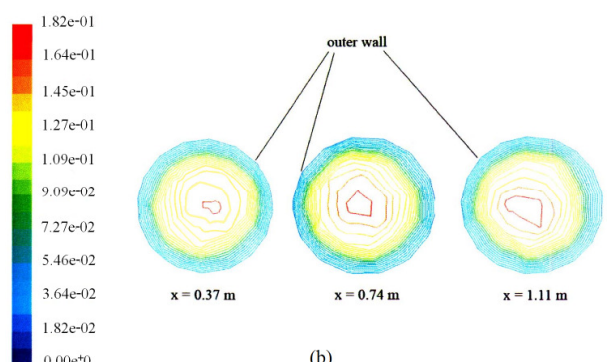

(b)
Figure 6. (a) velocity contour in the inner tube of parallel flow straight double tube heat exchanger
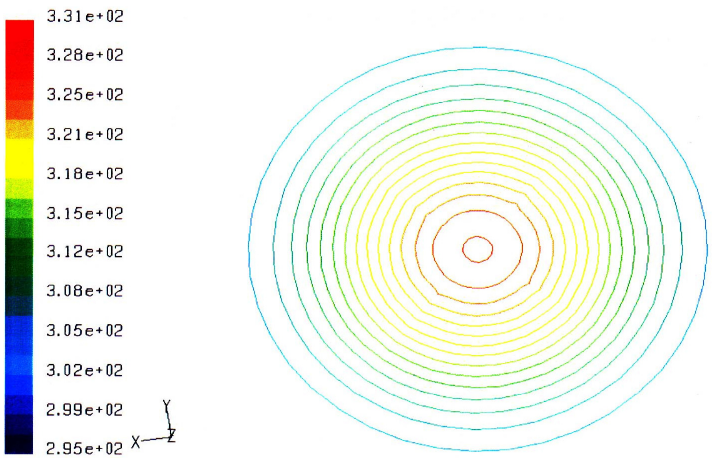

(a)

Figure 7. (a) Temperature contours in the straight double tube heat exchanger
Figure 6. (b) velocity contour in the inner tube of parallel flow helical double tube heat exchanger

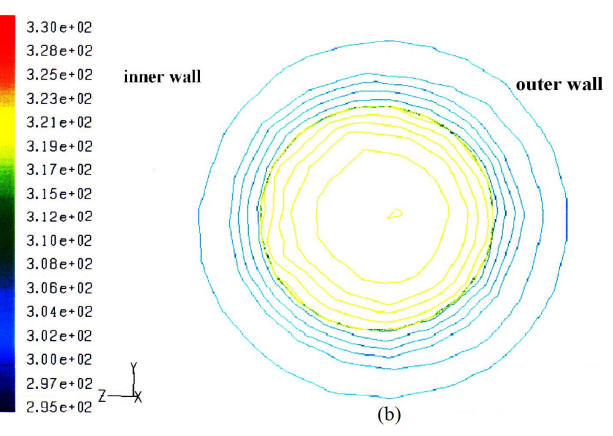

Figure 7. (b) Temperature contours in the helical double tube heat exchanger

\subsection{The effect of curvature ratio}

Curvature ratio is one of the most important parameters in helical double tube heat exchangers and it is presented by $d_{o} / 2 R$. Dean Number includes curvature ratio and it has to be noted that both parameters of do and $\mathrm{R}$ can change curvature ratio. Here, to avoid the variation of Reynolds number in annulus, just radius of the loop was changed. In Figure 8 it is observed that increasing of curvature ratio makes increasing of heat transfer coefficient significantly. It means that increasing of loop's radius makes drastically reducing of overall heat transfer.

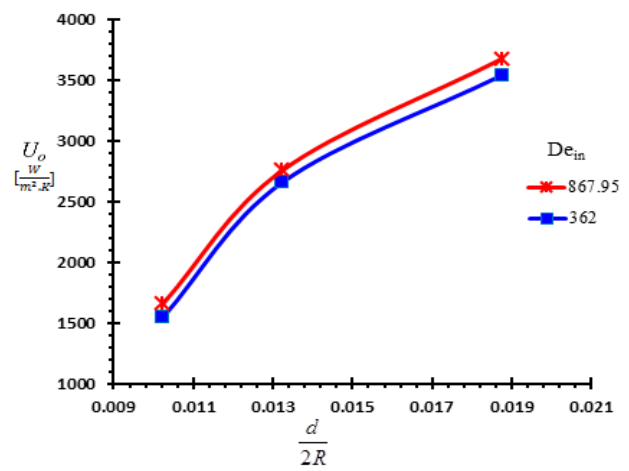

Figure 8. Influence of curvature ratio on overall heat transfer coefficient 
As the radius of the loops increases, fluid's torsion behavior approaches to linear behavior and also helical tube turns to straight tube. Because by increasing the radius of coils, the secondary flow effects due to centrifugal forces diminishes and flow of fluid through the coils tends to flow in a straight path and As a result, the friction coefficient decreases (Fig. 9).

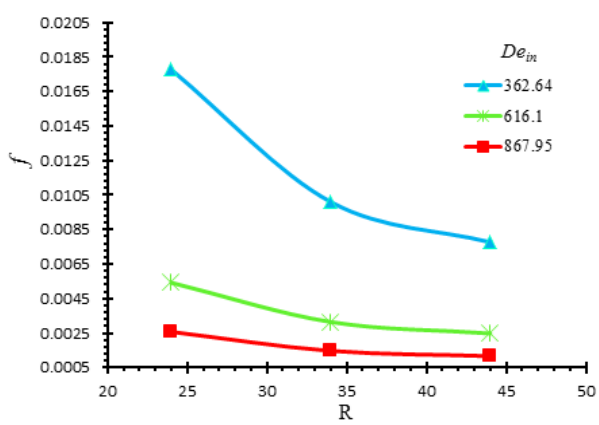

Figure 9. Influence of radius change on helical heat exchanger friction factor.

Figures 10-12 explain and illustrate the influence of radius changes on heat transfer coefficient. With these three-dimensional diagrams, the effects of Reynolds number through the inner tube and mass flow rate of the annulus on overall heat transfer coefficient can be compared simultaneously.

These figures indicate that, increase of annulus mass flow rate and internal Dean Number

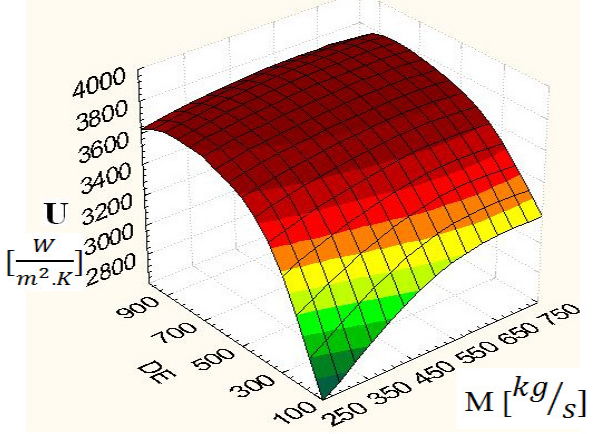

Figure 10. Influence of variation of annulus mass flow rate on overall heat transfer coefficient, $\mathrm{R}=24 \mathrm{~cm}$

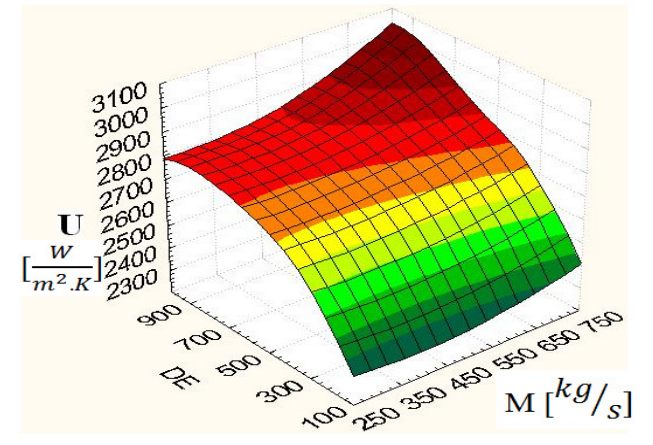

Figure 11. Influence of variation of annulus mass flow rate on overall heat transfer coefficient, $\mathrm{R}=34 \mathrm{~cm}$

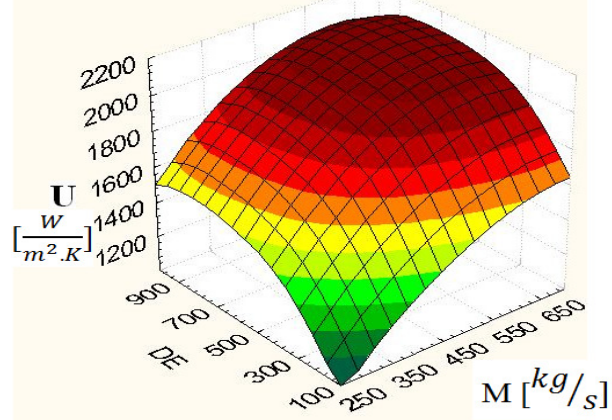

Figure 12. Influence of variation of annulus mass flow rate on overall heat transfer coefficient, $\mathrm{R}=44 \mathrm{~cm}$ increases the heat transfer coefficient between two fluids. 


\subsection{The Influence of pitch of coil}

Influence of tube's pitch on overall heat transfer coefficient of heat exchanger is surveyed in this section.

It must be noted that in this study, pitches of 1.6, 2, and 2.6 are used. Figure 13 indicates the influence of tube pitch size for different inner Dean Number on overall heat transfer coefficient in laminar flow.

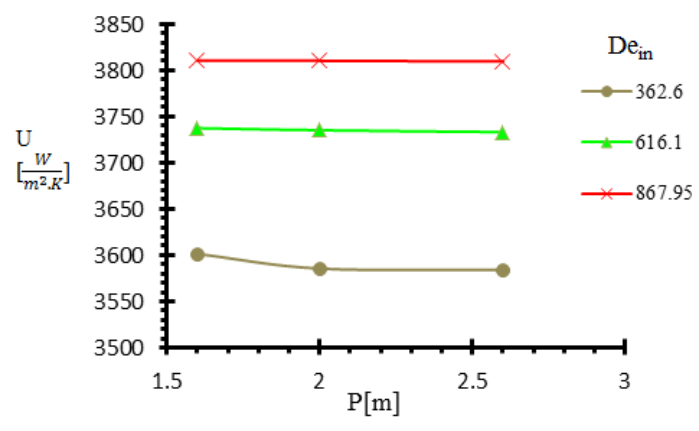

Figure 13. Influence of pitch increase on overall heat transfer coefficient

By increasing the pitch of coils, the torsion behaviour of flow diminishes and also, the heat transfer coefficient has been decreased. But, as can be seen from Figure 13, this influence is so negligible and it can be said that, the pitch of the loops doesn't have significant impact on heat transfer in helical exchangers.

\subsection{Influence of tube diameter change on heat transfer characteristics}

Another important parameter surveyed in this work, is the diameter of tubes used in heat exchanger. As it can be observed in Figure 14, increase of inner tube diameter and increase of inner Dean Number, makes Nusselt Number increase and consequently according to Nusselt Number's definition (Eq.15), heat transfer will increase.

$$
N u=\frac{h_{o} D}{k}
$$

The inner and outer heat transfer coefficients are usually obtained from the overall thermal resistance which is consisting of three resistances in series: the convective resistance in the inner surface, the conductance resistance of the pipe wall and the convective resistance on the outer surface by the equation 16 :

$$
\frac{1}{U}=\frac{A_{o}}{A_{i} h_{i}}+\frac{A_{o} \ln \left(d_{o} / d_{i}\right)}{2 \pi k L}+\frac{1}{h_{o}}
$$

where $d_{o}$ is the diameter of the outer tube; $d_{i}$ is the diameter of the inner tube; $k$ is the thermal conductivity of the wall and $\mathrm{L}$ is the length of the inner tube.

Heat transfer coefficients between the two tubes, $h_{o}$ and for the inner tube, $h_{i}$ were calculated using traditional Wilson plot technique [3, 6]. For the calculation of outer heat transfer coefficient in Eq. (15), the mass flow rate in the annulus side was kept constant; and assumed that the inner heat transfer coefficient is constant. The outer heat transfer coefficient was assumed to behave in the following manner with the fluid velocity in the tube side, $u_{o}$ : 


$$
h_{o}=C u_{o}^{n}
$$

Eq. (17) was placed into Eq. (16) and the values of the constant $\mathrm{C}$ and the exponent $\mathrm{n}$ were determined through the curve fitting.

Similar procedure was adopted for the calculation of inner heat transfer coefficient. On the other hand, from the figure 15 it can be noted that increasing the inner diameter of tubes also decreases the friction factor and consequently increases the pressure drop of flow through the tubes.

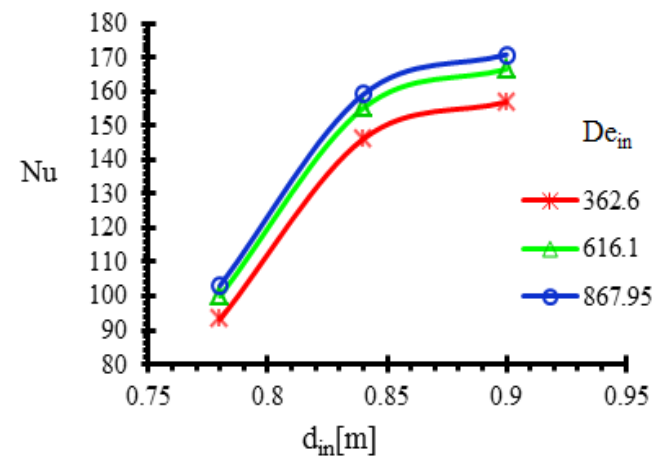

Figure 14. Influence of inner tube diameter on Nusselt Number

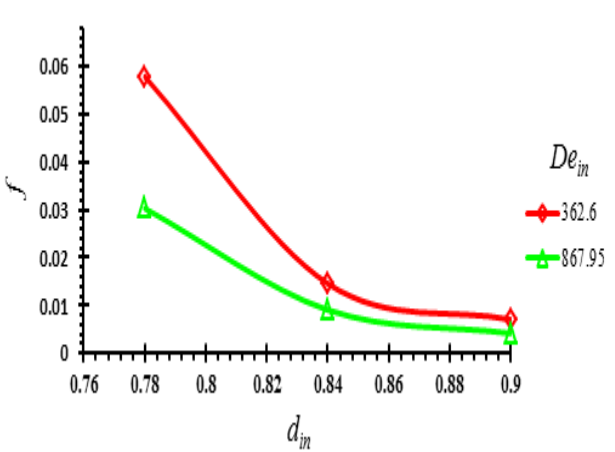

Figure 15. Influence of inner tube diameter on friction factor

\subsection{Influence of number of coils on overall heat transfer coefficient}

Most of the works done to investigate the effect of different parameters of helical heat exchangers are limited to one loop of tube. Therefore in this work the influence of other loops had been demonstrated. Increasing the number of coils is equal to lengthening the heat transfer path. So, according to the Figure 16, when the number of coils increases, the amount of the heat transfer coefficient decreases significantly, and in the number of coils larger than $n=6$, it approaches to a constant amount.

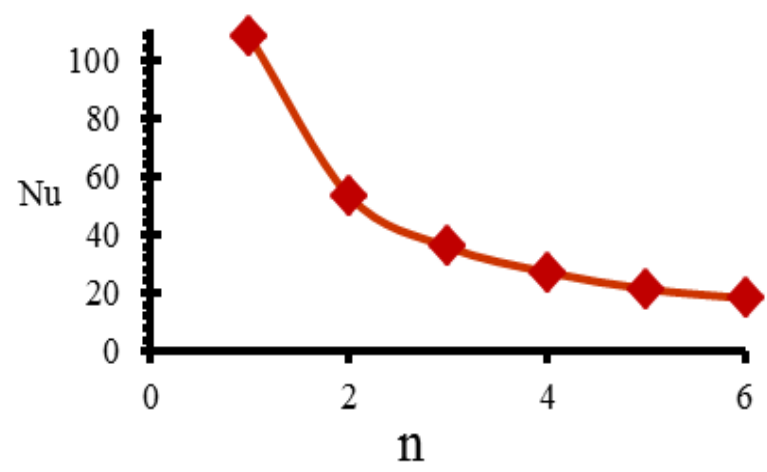

Figure 16. Influence of number of coils on overall heat transfer coefficient

Since the Prandtl number for water is larger than one $(\operatorname{Pr}>1)$, according to the Eqs. 18 and 19, the length of the thermal entrance $\left(\mathrm{L}_{\mathrm{T}}\right)$ will be greater than hydraulic entrance length $\left(\mathrm{L}_{\mathrm{H}}\right)$.

$L_{H}=0.058 \cdot \operatorname{Re}_{D} \cdot D$

$L_{T}=0.058 \cdot \operatorname{Re}_{D} \cdot D . \operatorname{Pr}$ 
Until fluid has not passed thermal entrance length, flow was developing and heat transfer coefficient decreases during the thermal development, and its least value will be occurred in fully developed region. Increasing the number of coils results in longer path, and this indicates that flow is thermally developed and in large numbers, the flow will be fully developed.

\section{Conclusions}

The present study has numerically conveyed heat transfer in helical double tube heat exchanger with variable fluid properties. In this investigation, at first by validation of obtained numerical results, with available experimental ones [15], it was shown that the present model is in a good agreement with experimental results of the literature. With surveying of different parameters, following results were obtained:

It is observed that, maximum velocity is located in central region of the inner tube because this region has maximum distance from the boundary layer.

Dean number is an important and effective parameter in helical double tube heat exchanger. By increasing of inner tube diameter, overall heat transfer coefficient of heat exchanger increases. It should be mentioned that, with increase of annulus mass flow rate (that leads to Dean number increase in annulus) rate of heat transfer will increase.

Nusselt Number has direct relevance with inner tube diameter and it means that increase of heat exchanger coil radius, leads to drastic decrease of overall heat transfer coefficient. It is argued that as coil radius increases, torsional behaviour of fluid approaches to linear behaviour and helical tube becomes straight tube.

Heat transfer coefficient was augmented considerably by increase of curvature ratio and this means along with coil radius, overall heat transfer reduces significantly. It can be argued that the more radius increments, torsional behaviour approaches towards linear behaviour.

Increasing the pitch of heat exchanger, leads to decrease of overall heat transfer coefficient of that. But this effect is negligible. So it can be concluded that, tube pitch is a parameter that doesn't have a great influence on analysis trend.

Increasing of number of coils means longer heat transfer path and observed decrease in Nusselt Number due to number of coils and this decrease in Nusselt Number due to number of coils indicates that flow is thermally developing and in large numbers the flow will be fully developed.

\section{References}

[1] Jain, M. and Jain, A., (2010) "Working vacation queueing model multiple types of server breakdown", Applied Mathematical Modelling, Vol. 34, No. 1, 1-13.

[2] Rennie T.J, Raghavan G.S.V. (2002) "Laminar parallel flow in a tube-in-tube helical heat exchange"; AIC2002 Meeting CSAE/SCGR Program, Saskatchwan.14-17.

[3] Rennie T.J, Raghavan G.S.V. (2006 )"Effect of fluid thermal properties on the heat transfer characteristics in a double-pipe helical heat exchanger", I.J. of Thermal Sciences, volume 45, 11581165.

[4] Kumar V, Saini S, Sharma M, Nigam K. D. P. (2006 )"Pressure drop and heat transfer study in tubein-tube helical heat exchanger", Chemical Engineering Science, volume61, 4403-4416.

[5] Lin C. X, Ebadian M. A. (2007) "Condensation heat transfer and pressure drop of R-134a in annular helicoidal pipe at different orientations", International Journal of Heat and Mass Transfer, Volume50, 4256-4264. 
[6] Kumar V, Faizee B, Mridha M, Nigam K. D. P. (2008) "Numerical studies of a tube-in-tube helically coiled heat exchanger", Chemical Engineering and Processing, volume 47, 2287-2295.

[7] Garrido D. C, Castelazo E. S, Hernandez J.A, Valladares O. G. Siqueiros J. Romero D.J. "Heat transfer of a helical double-pipe a vertical evaporator", Theoretical Analysis \&Experimental validation, App. Energy.

[8] Xiaowen Yi, Lee W.L. (2009) "The use of helical heat exchanger for heat recovery domestic watercooled air-conditioner", Energy conversion and management, volume50, 240-246.

[9] Xin RC, Awwad A, Dong Z.F, Ebadian M. A. (1997) "An Experimental study of single-phase and two-phase flow pressure drops in annular helicoidal pipes", International Journal of Heat and Fluid Flow, Volume18, 482-488,

[10] Petrakis M. A., Karahalios G. T. (1997) "Exponentially decaying flow in a gently curved annular pipe”, International Journal of None-linear Mechanics, 823-835.

[11] Petrakis M. A., Karahalios G. T. (1999) "Fluid flow behavior in a curved annular conduit", International Journal of None-linear Mechanics, Volume 34, 13-25.

[12] Han J. T, Lin C.X, Ebadian M. A. (2005) "Condensation heat transfer and pressure drop characteristics of R-134a in an annular helical pipe", International Communications in Heat \& Mass Transfer, volume 32, 1307-1316.

[13] Reza Beigzadeh, Masoud Rahimi. (2012) "Prediction of heat transfer and flow characteristics in helically coiled tubes using artificial neural networks", International Communications in Heat and Mass Transfer, volume 32, 1279-1285.

[14] Massimiliano Di Liberto, Michele Ciofalo. (2013) "A study of turbulent heat transfer in curved pipes by numerical simulation”, International Journal of Heat and Mass Transfer, 112-125.

[15] Gabriela Huminic, Angel Huminic. (2011) "Heat transfer characteristics in double tube helical heat exchangers using nanofluids", International Journal of Heat and Mass Transfer, 4280-4287.

[16] Rennie T.J, Raghavan G.S.V. (2005) "Experimental studies of a double pipe helical heat exchanger", Experimental Thermal and Fluid Science, 919-924.

[17] P.S. Srinivasan. (1968) "Pressure drop and heat transfer in coils", Chemical Engineering, 113-119.

[18] B. Xavier, T. Xavier, P. philippe, B. Philippe. "Comparison of tetrahedral and hexahedral meshe for organ finite element modelling": an application to kidney impact.

[19] Jayakumar J. S, Mahajani S. M, Mandal J. C, Vijayan P. K, Bhoi R. (2008) "Experimental and CFD estimation of heat transfer in helically coiled heat exchangers", Chemical Engineering Research and Design; 86:221-232.

[20] D. Colorado, E. Santoyo, J. Hernandez, O. Garcia, J. Siqueiros, D. Juarez; (2009) "Heat Transfer of a helical double-pipe vertical evaporator", Theoretical analysis and Experimental validation, Applied Energy 86 (7-8) 1144-1153. 